\section{Knowledge, attitudes, and self care practices associated with age related eye disease in Australia}

\author{
P M Livingston, C A McCarty, H R Taylor
}

not have regular and timely eye examinations to ensure early diagnosis and treatment. Appropriate eye health education may encourage preventative eye health care, creating an avenue for early diagnosis and treatment before visual loss has occurred.

The Visual Impairment Project ${ }^{1}$ found that half of the population sample had not accessed either an optometrist or ophthalmologist within the past 2 years. ${ }^{2}$ With limited resources available in the health budget, preventive medicine offers an economically and socially acceptable alternative to preventable visual impairment. Implementation of health education that encourages people in the community towards early detection and treatment, may identify those individuals who would otherwise be unaware or unable to obtain recommended examinations and treatment. The knowledge of an individual is an important predictor of behavioural change in the treatment of hypertension $^{3}$ and cancer, ${ }^{4}$ and in secondary prevention and early diagnosis, such as alcohol abuse. $^{5}$

An exploration of knowledge and attitudes held, and the self care practices undertaken by the community, can aid in the effective promotion of preventive approaches to eye health care. However, despite its significance, little information is available on knowledge, attitudes, and self care practices (KAP) associated with age related eye disease and the elderly population.

The aims of this study were to investigate the knowledge of age related eye disease, attitudes to blindness prevention and treatment, and self care practices in a population based sample.

(Br F Ophthalmol 1998;82:780-785)

To reduce visual impairment in an aging community, timely eye examinations and appropriate treatment are necessary. Yet many people in the "at risk population" group frequently do

\section{Method}

The Visual Impairment Project (VIP) was a population based survey of noninstitutionalised residents aged 40 years or more in urban and rural settings. The detailed methodology has been reported elsewhere. ${ }^{67}$ districts were randomly selected from the Melbourne Statistical Division and four rural clusters in rural Victoria. ${ }^{8} \mathrm{~A}$ door to door household census was taken to identify all eligible people - that is, those aged 40 years or more in the calendar year of examination and who had lived at that address for 6 months or more. Information about household characteristics and basic demographic data was collected during a brief interview conducted at the household. Eligible individuals were invited to a local examination site for a structured interview and standardised ophthalmic examination. The project had the approval of In brief, nine pairs of adjacent census collector \begin{abstract}
knowledge of common age related eye disease also considered blindness treatment to be the highest priority compared with other diseases. People with a previous diagnosis of age related eye disease, older people, females, people with correct knowledge of common eye diseases, and those who spoke English at home were significantly more likely to be under eye care. No interaction was found between knowledge and positive attitudes to self care practices.

Conclusion-These data show that there is a large gap in the public's knowledge and understanding of eye disease that will need to be understood for eye health promotion activities.
\end{abstract}

Accepted for publication 22 January 
Table 1 Awareness and knowledge of age related eye disease in the population sample

\begin{tabular}{lll}
\hline Disease type & Aware of eye disease* & $\begin{array}{l}\text { Correct knowledge of eye diseaset } \\
(n=3185)\end{array}$ \\
\hline Cataract & $2801 / 3028(92 \%)$ & $2379(74 \%)$ \\
Glaucoma & $2664 / 3131(79 \%)$ & $623(19 \%)$ \\
AMD & $151 / 3166(5 \%)$ & $64(2 \%)$ \\
\hline
\end{tabular}

^People who were previously diagnosed with the age related eye disease were not asked their awareness of that particular disease.

†All people who self reported an awareness of the disease or who were previously diagnosed with the condition were asked to describe the disease in question.

the Royal Victorian Eye and Ear Hospital human research ethics committee.

To ascertain the overall level of knowledge in the community, participants from the first five sample areas of the Melbourne VIP and all of the rural participants were asked additional questions on their knowledge of the common age related eye conditions, cataract, age related macular degeneration (AMD) and glaucoma. The sample was limited to the first five sample areas in metropolitan Melbourne as sufficient power has been achieved to establish prevalence with narrow $95 \%$ confidence intervals. ${ }^{9}$

A subset of this population sample was asked questions on their attitudes towards the prevention and treatment of blindness knowledge of common eye conditions.

\section{Definitions}

AWARENESS OF AGE RELATED EYE DISEASE

People who were not previously diagnosed with one or more of the three conditions were asked if they had heard of cataract, glaucoma, and AMD (yes/no).

PREVIOUS DIAGNOSIS OF AGE RELATED EYE DISEASE

A respondent was classified as being previously diagnosed with cataract, $\mathrm{AMD}$, or glaucoma, if they self reported that a doctor had diagnosed then as having any of the three eye conditions. Previous diagnosis was assessed as the potential to confound the relation between knowledge, attitudes, and self care practices.

FAMILY HISTORY OF AGE RELATED EYE DISEASE Participants were questioned on their family's history of age related eye disease, cataract, glaucoma, and AMD. Family members comprised parents and siblings.

\section{CORRECT KNOWLEDGE OF EYE DISEASE}

During the interview, respondents previously diagnosed as having an eye disease, or who indicated that they were aware of the disease in question, were asked to describe it. Acceptable,

Table 2 Univariate and multivariate logistic regression assessing the association between correct knowledge of age related eye disease and sociodemographic factors

\begin{tabular}{lll}
\hline Factors & $\begin{array}{l}\text { Univariate odds } \\
\text { ratio (95\% CI) }\end{array}$ & $\begin{array}{l}\text { Multivariate odds } \\
\text { ratio (95\% CI) }\end{array}$ \\
\hline Age (10 years) & $1.12(1.05-1.20)$ & $1.01(1.004-1.02)$ \\
Sex (females) & $1.46(1.24-1.72)$ & $1.50(1.26-1.79)$ \\
Born in Australia & $2.23(1.89-2.67)$ & $1.24(0.99-1.57)$ \\
English spoken at home & $6.33(4.88-8.13)$ & $4.48(3.25-6.17)$ \\
Positive family history & $1.19(0.92-1.54)$ & $\mathrm{ns}$ \\
Education (secondary/trade/tertiary) & $2.15(1.82-2.55)$ & $1.65(1.37-1.99)$ \\
Location (rural) & $1.15(0.98-1.36)$ & $\mathrm{ns}$ \\
Previous diagnosis of cataract, AMD, or glaucoma & $1.26(0.95-1.67)$ & $\mathrm{ns}$ \\
Last visit to eye practitioner (<2 years) & $1.36(1.15-1.60)$ & $1.34(1.11-1.61)$ \\
\hline
\end{tabular}

correct definitions were provided by co-author (HRT) and are presented below.

Cataract: lens opacity; film over the eye; causes vision loss/blindness; affects the elderly; causes blurred vision;

Macular degeneration: problem related to the retina; causes vision loss/blindness; central focusing problem; affects the elderly;

Glaucoma: pressure problem related to the eye; causes vision loss/blindness; causes tunnel vision; affects the elderly; problem at the back of the eye.

People who provided knowledge on one or more of the three eye conditions were compared with those who did not provide any information on one or more of the three eye conditions.

\section{ATtiTUdES}

Participants were asked additional questions on their attitudes on the prevention and treatment of blindness. Responses were dichotomised into those who reported blindness prevention or treatment and support as their highest priority compared with the other conditions and those who did not.

SELF CARE PRACTICES

All participants were asked the year of their last visit to an eye practitioner-that is, either an ophthalmologist or optometrist. The period of the most recent visit to an eye service was grouped as 2 years or less, 3 or more years, or never.

The following categories were used: age was grouped into decades, language spoken at home as English or other language; country of birth as Australia or other; family history as a family member with a diagnosis of cataract, AMD, glaucoma, or none; location as rural compared with urban and education level was grouped into primary or secondary, trade, or tertiary.

For people who could not speak English, family members or employed interpreters translated the questions and responses given.

Participants were not prompted to possible responses. Data were recoded and analysed using SAS (Cary, NC, USA). Statistical methods included the $\chi^{2}$ test for comparing groups; odds ratios and $95 \%$ confidence intervals were calculated using logistic regression techniques. The results of the analyses were considered statistically significant when $p<0.05$.

\section{Results}

A total of 1711 people participated in eye examinations in the first five sample areas of the Melbourne VIP and 1473 participants from the four sample areas of rural Victoria. This represented an $89 \%$ participation rate from the total of 5520 residents enumerated in the private household census. In metropolitan Melbourne, although non-English speaking people were less likely to participate (OR: 0.61; CI: 0.48-0.77), there were no other significant differences between participants and nonparticipants and between participants and the Melbourne and Australian populations. ${ }^{10}$ In rural Victoria, there were no significant 
Table 3 Knowledge by significant predictors of age related eye disease

\begin{tabular}{llllll}
\hline $\begin{array}{l}\text { Knowledge of age related eye } \\
\text { disease }\end{array}$ & Sex (female) & Age $<70$ years & $\begin{array}{l}\text { English spoken at } \\
\text { home }\end{array}$ & $\begin{array}{l}\text { Recent visit to eye } \\
\text { practitioner } \\
\text { (<2 years) }\end{array}$ & $\begin{array}{l}\text { Education } \\
>\text { primary education }\end{array}$ \\
\hline Cataract $(\mathrm{n}=2379)$ & $1329(56 \%)$ & $1875(79 \%)$ & $2270(96 \%)$ & $1495(63 \%)$ & $1729(73 \%)$ \\
Glaucoma (n=623) & $377(61 \%)$ & $469(75 \%)$ & $610(98 \%)$ & $429(69 \%)$ & $480(77 \%)$ \\
AMD $(\mathrm{n}=64)$ & $44(69 \%)$ & $43(67 \%)$ & $63(98 \%)$ & $55(86 \%)$ & $44(69 \%)$ \\
\hline
\end{tabular}

differences between participants and nonparticipants. The participants ranged in age from 40 to 103 years and $53 \%$ were female.

A total of $2443(77 \%)$ people reported that they were aware of one or more of the three eye conditions. The majority of people provided knowledge of cataract (74\%) (Table 1). Many respondents confused glaucoma with other eye conditions: $10 \%$ described it as cataract or a growth over the eye; $4 \%$ described it as trachoma, and $1 \%$ linked it with diabetes; 3\% of people who stated an awareness of AMD confused the disease with cataract. Seven per cent of people who had knowledge of cataract, $6 \%$ who had knowledge of glaucoma, and $11 \%$ of people who had knowledge of AMD provided general information on the diseasesthat is, the condition causes blindness/vision loss or affects the elderly.

To evaluate the association of specific sociodemographic characteristics among those who provided correct knowledge about age related eye disease, several regression models were constructed (Table 2).

A multivariate logistic regression model was constructed using all of the significant factors from the univariate analyses (Table 2). When the individual variables were adjusted for, the significant predictors of correct knowledge of eye disease were: age (younger), sex (females), higher levels of formal education, a recent visit to an eye practitioner, and English spoken at home. Contingency table analyses are presented (Table 3).

A subsample of 1557 participants was asked questions to determine their attitudes to the prevention of total blindness. Overall, $25 \%$ of the sample indicated that they would prevent total blindness first compared with cancer of the bowel (36\%), Alzheimer's disease (24\%), schizophrenia $(8 \%)$, or heart disease $(7 \%)$. The univariate analysis assessing the association between those who gave blindness prevention their first priority and specific sociodemographic factors highlighted that older people were less likely to regard blindness as a high priority (Table 4 ).

The subsample of participants were then asked their attitudes on the treatment of total blindness. Nearly two thirds $(60 \%)$ of the sample reported that they would treat total blindness first compared with paralysis on one side of the body as a result of stroke $(16 \%)$, loss of speech as a result of a stroke $(15 \%)$, total deafness (4\%), and amputation of an arm $(4 \%)$. A univariate analysis assessed the characteristics associated with attitudes towards blindness treatment and a multivariate analysis performed (Table 4). The significant predictors of positive attitudes towards blindness treatment were a recent visit to an eye practitioner (within the past 2 years); younger age, correct knowledge of eye disease, and speaking English at home (Table 4).

Contingency table analyses indicated that $57 \%$ of people who spoke English at home considered blindness treatment a first priority compared with $30 \%$ of people who spoke another language at home; $58 \%$ of people with correct knowledge specified treatment as the priority compared with $48 \%$ of people who did not have any knowledge of age related eye disease; and $47 \%$ of people over the age of 70 considered visual impairment the important priority compared with $53 \%$ of people under the age of 70 .

The third stage of the analysis was to determine factors that may influence self care practices or the regular utilisation of eye services. The distribution of optometric and ophthalmic service utilisation was similar in both areas (data not shown) so we grouped the urban and rural cohorts together. Overall, 1953 (61\%) of the population sample had visited an eye practitioner, either an ophthalmologist or optometrist within the past 2 years compared with $1232(39 \%)$ who had done so 3 or more years ago or never.

Again, a multivariate regression model was constructed after the univariate analyses (Table

Table 4 Univariate and multivariate regression assessing the association between positive attitudes - that is, people who reported blindness prevention or treatment as their highest priority, with sociodemographic factors

\begin{tabular}{llll}
\hline & \multicolumn{2}{l}{ Univariate OR (95\% CI) } & Multivariate OR (95\%, \\
\cline { 2 - 4 } Sociodemographic factors & $\begin{array}{l}\text { Prevention of total } \\
\text { blindness as highest } \\
\text { priority }\end{array}$ & $\begin{array}{l}\text { Treatment of total } \\
\text { blindness highest } \\
\text { priority }\end{array}$ & $\begin{array}{l}\text { Clindness as highest } \\
\text { priority }\end{array}$ \\
\hline Age group (10 years) & $1.01(1.00-1.02)$ & $1.02(1.008-1.025)$ & $1.01(1.005-1.02)$ \\
Sex (females) & $1.02(0.81-1.29)$ & $1.26(1.01-1.57)$ & $1.13(0.82-1.55)$ \\
Born in Australia & $1.06(0.93-1.21)$ & $1.03(0.95-1.12)$ & $\mathrm{ns}$ \\
English spoken at home & $1.13(0.56-2.26)$ & $3.04(1.57-5.88)$ & $2.59(1.33-5.08)$ \\
Positive family history & $1.52(0.57-4.03)$ & $1.76(0.70-4.41)$ & $\mathrm{ns}$ \\
Education (secondary/trade/tertiary) & $1.01(0.78-1.25)$ & $1.28(1.11-1.72)$ & $1.25(1.02-1.75)$ \\
Location (rural) & $1.16(0.72-1.87)$ & $1.22(0.94-1.57)$ & $\mathrm{ns}$ \\
Previous diagnosis of cataract, AMD, or glaucoma & $1.14(0.79-1.63)$ & $1.34(0.95-1.78)$ & $\mathrm{ns}$ \\
Last visit to eye practitioner <2 years & $1.14(0.87-1.45)$ & $1.12(0.90-1.41)$ & $\mathrm{ns}$ \\
Knowledge of cataract, AMD, or glaucoma & $1.21(0.90-1.62)$ & $1.48(1.14-1.72)$ & $1.34(1.02-1.75)$ \\
\hline
\end{tabular}


Table 5 Multivariate regression assessing the association between self care practices - that $i$, visit to an eye practitioner within the past 2 years and sociodemographic factors

\begin{tabular}{ll}
\hline Sociodemographic factors & Odds ratio (95\% CI) \\
\hline Age group (10 years) & $1.03(1.01-1.035)$ \\
Sex (female) & $1.16(1.01-1.35)$ \\
Knowledge of cataract, AMD, or glaucoma & $1.33(1.01-1.58)$ \\
English spoken at home & $1.37(1.18-1.93)$ \\
Previous diagnosis of cataract, AMD, or glaucoma & $2.84(2.06-3.92)$ \\
\hline
\end{tabular}

5). The significant predictors of self care practices were a previous diagnosis of an age related eye disease, age, sex, knowledge of age related eye disease, and English spoken at home. When the potential confounding variable previous diagnosis of cataract, glaucoma, and AMD was removed from the multivariate analysis, there was found to be no significant confounding effect whatsoever.

Contingency table analyses indicated that $85 \%$ of people with a previous diagnosis of cataract, AMD or glaucoma visited an eye practitioner within the past 2 years compared with $58 \%$ who did not have a previous diagnosis; $53 \%$ of people over the age of 60 years visited an eye practitioner in the past 2 years compared with $38 \%$ of people 3 or more years ago; $63 \%$ of females had visited an eye practitioner in the past 2 years compared with $59 \%$ of males; and $62 \%$ of people who spoke English at home had a recent visit to an eye practitioner compared with $52 \%$ of people who spoke another language.

The final stage of the analysis was to determine whether a combination of knowledge of any of the age related eye disease and positive attitudes towards the prevention and treatment of total blindness would influence self care practices. However, an interaction between correct knowledge and attitudes with a recent visit to an eye practitioner was not found (OR: 1.15; 95\% CI: 0.97-1.37) (Fig 1).

\section{Discussion}

This investigation examined the knowledge, attitudes, and self care practices of individuals in an urban population sample. To our knowledge, no other study has investigated the influence of location (urban compared with rural communities) or the potential role of family history of age related eye disease and KAP. The study was undertaken to identify information that could enhance a public health campaign designed to reduce the prevalence and incidence of the three most common age related eye diseases. The results may help target specific groups within the community.
Few studies have investigated the relation between KAP and age related eye disease. ${ }^{11-13}$ The results of the current study indicated that the majority of people had correct knowledge of one or more of the three eye conditions specified; however, this was mainly attributable to respondents providing a lay description of cataract - that is, "a film over the eye". There were serious deficiencies in respondent knowledge of both glaucoma and AMD, with $20 \%$ of respondents confusing glaucoma and AMD with other eye conditions. This lack of knowledge is consistent with other studies conducted in Germany and the United States. ${ }^{10}{ }^{11}$

In this study, younger people, females, those with higher levels of education and those speaking English at home were more likely to have a better understanding of age related eye disease. Similar predictors of correct knowledge have been demonstrated in other KAP studies conducted both in other health areas as well as eye disease..$^{10}{ }^{14}$ Pronzato et al ${ }^{14}$ also found that women and patients with a higher education level were more knowledgeable about their disease. Michielutte et al ${ }^{12}$ found that females and people who reached higher levels of education had the highest levels of knowledge of glaucoma and diabetes. Researchers of the Lighthouse National Survey ${ }^{10}$ found that the youngest, wealthy, and more educated respondents were more likely to be informed about visual impairment.

Older people did not appear to consider blindness prevention as a particularly high priority. This may have been attributable to the design of the questions themselves. The choices offered in the question on the prevention of disease included mortality-type conditions such as heart disease and cancer of the bowel as well as morbidity-type conditions, such as total blindness. Verbrugge et al ${ }^{15}$ analysed the impact of several conditions on adults and found that life threatening conditions such as heart disease and malignant neoplasms received greater community prominence despite the fact that non-fatal conditions such as visual impairment, arthritis, and hearing impairment had a significant long term impact on both the individual and the community.

Other studies ${ }^{101617}$ have indicated that knowledge of a condition or disease can positively influence self care practices. In this study, knowledge of age related eye disease appeared to influence positive self care practices.

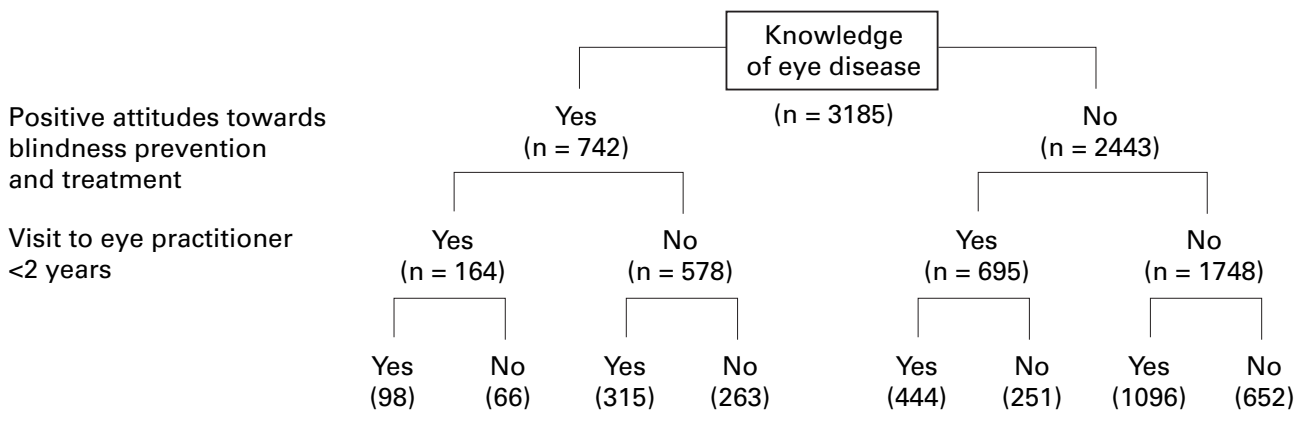

Figure 1 Tree of knowledge and positive attitudes and their association with use of eye services. 
Women were more likely to participate in regular eye health care. This is consistent with the previous reports of women's overall perception of health care and the propensity for women to seek out preventive health care compared with men. ${ }^{18}$ Interestingly, people who had correct knowledge of age related eye disease were more likely to participate in regular utilisation of eye services; however, a combination of both correct knowledge and attitudes that considered prevention and treatment of total blindness as the highest priority did not appear to interact with self care practices. Further research is required to establish the reasons why people participate in ophthalmic eye services on a regular basis. It could be that fear of going blind or losing vision predicts people's behaviour. Alternatively, it may be the individual's income predicts behaviour. Constraints on income may limit self care practices.

There are limitations to this study that need to be considered when interpreting the results. Firstly, self report measures can result in recall bias; however, in any study, inaccuracies are unavoidable when self report measures form part of the study design. In addition, the concepts that we were attempting to assess may be influenced by current prevalence rates of disease in the community and state of the art in disease definition over time. With an aging community, people are more likely to develop age related eye conditions; and with new diagnostic equipment, we are more likely to diagnose the diseases earlier.

Notwithstanding these limitations, the results of this study suggest that there is limited knowledge among those at greatest risk of suffering from vision loss and visual impairment as a result of age related eye disease. Educating the community on glaucoma, AMD, and to a lesser extent cataract will be an important first component in the promotion of preventative ophthalmic care towards a reduction of visual impairment in the community. Redressing the lack of knowledge associated with common eye disease poses a challenge for public health professionals. To maximise their effectiveness, innovative information programmes must be focused on groups that are at greatest risk of losing their vision. In the first instance, information programmes that highlight the consequences of age related eye disease on the individual, the benefits of regular eye examinations, and appropriate treatment could be undertaken by general practitioners. General practitioners are important agents of health behaviour change as they have the opportunity to affect, at a population level, at least $80 \%$ of the population in Australia each year. ${ }^{19}$ General practitioners, therefore, are well placed to target the aging community, particularly males, people from non-English speaking backgrounds, and those with limited formal education.

The authors wish to acknowledge the contributions of the following people: Dr Charles Guest, Ms Cara Jin, field staff from the Roy Morgan Research Centre, and other staff who contributed to the VIP. The Visual Impairment Project is supported in part by the Victorian Health Promotion Foundation, the Ansell Ophthalmology Foundation, the Dorothy Edols
Estate, the Jack Brockhoff Foundation, and the National Health Estate, the Jack Brockhoff Fou
$\&$ Medical Research Council.

\section{Appendix 1}

KNOWLEDGE

Have you ever been told that you have glaucoma?

If no,

Have you ever heard of the eye condition glaucoma?

If yes to either question,

How would you describe it? (record all mentioned)

Have you ever been told that you have macular degeneration?

If no,

Have you ever heard of the eye condition macular degeneration?

If yes to either question,

How would you describe it? (record all mentioned)

Have you ever been told that you have cataracts?

If no,

Have you ever heard of the eye condition cataract?

If yes to either question,

How would you describe it (record all mentioned)

ATTITUDES

If it were possible to prevent only one of the following diseases, which of these diseases would you prevent first? (hand card to respondent or read out)

Condition

Rank

Cancer of the bowel or gut

Rank

Schizophrenia (a mental disorder)

Heart disease

Total blindness

Alzheimer's disease

Can't say

Which would you prevent second? third? fourth?

If it were possible to provide treatment and support for one of the following disabilities, which of these disabilities would you provide treatment and support for first? (hand card to respondent or read out)

Condition

Rank

Paralysis on one side of the body as a result of a

stroke

Loss of speech as a result of a stroke

Total deafness

Total blindness

Amputation of an arm

Can't say

Which would you provide treatment and support for the second? third? fourth?

SELF CARE PRACTICES

Have you ever been to an optometrist (a person who checks to see if you need to wear glasses and may supply them)?

Yes Record year of last visit

Have you ever been to an ophthalmologist, that is a medical doctor (a doctor who has specialsied in eye disease)? 
1 Taylor HR, Livingston PM, Stanislavsky YL, et al. Visual impairment in Australia: distance and near visual acuity and visual field findings of the Melbourne Visual

2 Impairment Project. Am f Ophthalmol 1997;123:328-37. care services by people with diabetes: the Melbourne Visual Impairment Project. Br f Ophthalmol 1998;82:410-$$
14 .
$$

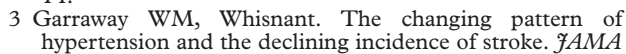
1987;258:214-7.

4 Worden JK, Solomon LS, Flynn BS, et al. A community wide program in breast self-examination training and maintenance. Prev Med 1990;19:254-69.

$5 \mathrm{Kraft}$ DP. The prevention and treatment of alcohol problems on a college campus. F Alcohol Drug Ed 1988;34: 37-51.

6 Livingston PM, Carson CA, Stanislavsky YL, et al. Methods for a population-based study: the Melbourne Visual Impairment Project. Ophthalmic Epidemiol 1994;1:139-48

7 Livingston PM, Guest CS, Bateman A, et al. Cost effectiveness of recruitment methods in a population-based ness of recruitment methods in a population-based epidemiological study. Aust f Pub Health 1994;18:314-18. Castles I. How Australia takes a census.

9 Lwanga SK, Lemeshow S. Sample size determination in health studies. A practical manual. Geneva: WHO, 1991.

10 Livingston PM, Lee SE, McCarty CA, et al. A comparison of participants with non-participants in a population-based epidemiologic study: the Melbourne Visual Impairment Project. Ophthalmic Epidemiol 1997;4:73-81.

11 Pfeiffer N, Krieglestein GK. Knowledge about glaucoma in the population. Invest Ophthalmol Vis Sci 1993;s1192.

12 Michielutte R, Diseker RA, Stafford CL, et al. Knowledge of diabetes and glaucoma in a rural North Carolina community. F Commun Health 1984;9:269-84.

13 Richards F, Klein RE, Gonzales-Peralta C, et al. Knowledge, attitudes and perceptions (KAP) of onchocerciasis: a survey among residents in an endemic area in Guatemala targeted for mass chemotherapy with ivermectin. Soc Sci Med 1991;11:1275-81.

14 Pronzato P, Bertelli G, Losardo P, et al. What do advanced cancer patients know of their disease? A report from Italy. Support Care Cancer 1994;2:242-4.

15 Verbrugge LM, Patrick DL. Seven chronic conditions: their impact on US adults' activity levels and use of medical services. Am f Public Health 1995;85:173-82.

16 Rosenstock I. Why people use health services. Millbank Mem Fund Q 1966;44:94-124.

17 Setter N. Subjective probability and decisions under uncertainty. Psychol Rev 1959;66:150.

18 Green LW, Kreyter MW. Health promotion planning. An educational and environmental approach. 2nd ed. USA: Mayfield Publishing Co, 1992.

19 Australian Bureau of Statistics. The Australian health survey 1992-3: doctors' consultations. Canberra: Australian Bureau of Statistics, 1995. 